SUPPORTING INFORMATION

\title{
Thermal Decomposition Mechanism of Disilane
}

\author{
Kazumasa Yoshida, ${ }^{\dagger}$ Keiji Matsumoto, ${ }^{\dagger}$ Tatsuo Oguchi, ${ }^{\ddagger}$ Kenichi Tonokura, ${ }^{*},{ }^{\dagger,}{ }^{\$}$ and Mitsuo Koshi ${ }^{\dagger}$ \\ ${ }^{\dagger}$ Department of Chemical System Engineering, School of Engineering, The University of Tokyo, 7-3-1 Hongo, \\ Bunkyo-ku, Tokyo 113-8656, Japan \\ ${ }^{\ddagger}$ Department of Ecological Engineering, Toyohashi University of Technology, Toyohashi 441-8580, Japan \\ \$ Present address: Environmental Science Center, The University of Tokyo, 7-3-1 Hongo, Bunkyo-ku, Tokyo \\ 113-0033, Japan
}

Gas phase rate constants are given in Table S1. Surface phase rate constants are given in Table S2. Concentrations of $\mathrm{Si}_{2} \mathrm{H}_{6}, \mathrm{Si}_{3} \mathrm{H}_{8}$, and $\mathrm{Si}_{2} \mathrm{H}_{4}$ species measured at $\mathrm{T}=720 \mathrm{~K}$ and $\mathrm{P}=36.5$ Torr in this study are given in Table $3 \mathrm{~S}$. Concentrations of $\mathrm{Si}_{2} \mathrm{H}_{6}, \mathrm{Si}_{3} \mathrm{H}_{8}, \mathrm{Si}_{2} \mathrm{H}_{4}, \mathrm{Si}_{4} \mathrm{H}_{10}$, and $\mathrm{SiH}_{4}$ and branching ratios of $\mathrm{SiH}_{4}$ to $\mathrm{Si}_{3} \mathrm{H}_{8}$ at $\mathrm{T}=720 \mathrm{~K}$ and $\mathrm{P}=36.5$ Torr predicted by the MSG1 model are given in Table 4S. 
TABLE S1: Gas phase rate constants. ${ }^{\text {a }}$ Forward and/or reverse rate constants are expressed in the form $k_{\mathrm{f}}=A T^{\square}$ $\exp \left(-E_{\mathrm{a}} / R T\right)^{\mathrm{b}}$

\begin{tabular}{|c|c|c|c|c|c|c|c|}
\hline \multirow[t]{2}{*}{ No. } & \multirow[t]{2}{*}{ Reaction } & \multicolumn{3}{|l|}{36.5 Torr } & \multicolumn{3}{|l|}{ 35.0 Torr } \\
\hline & & A & $\beta$ & $\mathrm{E}_{\mathrm{a}}$ & A & $\beta$ & $\mathrm{E}_{\mathrm{a}}$ \\
\hline \multirow[t]{2}{*}{1} & $\mathrm{Si}_{2} \mathrm{H}_{6}(+\mathrm{M})=\mathrm{SiH}_{2}+\mathrm{SiH}_{4}(+\mathrm{M})$ & $5.27 \mathrm{E}+12$ & 0.0 & 45765.0 & $5.02 \mathrm{E}+12$ & 0.0 & 45724.7 \\
\hline & (reverse reaction) & $1.32 \mathrm{E}+11$ & 0.0 & -6897.7 & $1.26 \mathrm{E}+11$ & 0.0 & -6926.3 \\
\hline \multirow[t]{2}{*}{2} & $\mathrm{Si}_{2} \mathrm{H}_{6}(+\mathrm{M})=\mathrm{H}_{3} \mathrm{SiSiH}+\mathrm{H}_{2}(+\mathrm{M})$ & $4.81 \mathrm{E}+10$ & 0.0 & 45549.1 & $4.58 \mathrm{E}+10$ & 0.0 & 45514.0 \\
\hline & (reverse reaction) & $1.31 \mathrm{E}+08$ & 0.0 & -11200.6 & $1.23 \mathrm{E}+08$ & 0.0 & -11235.9 \\
\hline \multirow[t]{2}{*}{3} & $\mathrm{SiH}_{2}+\mathrm{SiH}_{4}(+\mathrm{M})=\mathrm{H}_{3} \mathrm{SiSiH}+\mathrm{H}_{2}(+\mathrm{M})$ & $9.55 \mathrm{E}+12$ & 0.0 & 2140.6 & $9.44 \mathrm{E}+12$ & 0.0 & 2116.3 \\
\hline & (reverse reaction) & $1.20 \mathrm{E}+12$ & 0.0 & -2041.6 & $1.19 \mathrm{E}+12$ & 0.0 & -2055.9 \\
\hline \multirow[t]{7}{*}{4} & $\mathrm{SiH}_{4}(+\mathrm{M})=\mathrm{SiH}_{2}+\mathrm{H}_{2}(+\mathrm{M})$ & & & & & & \\
\hline & High-pressure limit & $3.12 \mathrm{E}+09$ & 1.7 & 54710 & & & \\
\hline & Low-pressure limit & $5.21 \mathrm{E}+29$ & -3.5 & 57550 & & & \\
\hline & Troe parameters : & & & & & & \\
\hline & $a=-0.4984, T^{* * *}=888.3, T^{*}=209.4$ & & & & & & \\
\hline & Enhanced third-body efficiensies : & & & & & & \\
\hline & $\mathrm{SiH}_{4}=4, \mathrm{Si}_{2} \mathrm{H}_{6}=4$ & & & & & & \\
\hline \multirow[t]{7}{*}{5} & $\mathrm{Si}_{3} \mathrm{H}_{8}(+\mathrm{M})=\mathrm{SiH}_{2}+\mathrm{Si}_{2} \mathrm{H}_{6}(+\mathrm{M})$ & & & & & & \\
\hline & High-pressure limit & $6.97 \mathrm{E}+12$ & 1 & 52677 & & & \\
\hline & Low-pressure limit & $1.73 \mathrm{E}+69$ & -15 & 60491 & & & \\
\hline & Troe parameters : & & & & & & \\
\hline & $a=-3.47 \mathrm{E}-05, T^{* * *}=442.0, T^{*}=2414$ & 128.3 & & & & & \\
\hline & Enhanced third-body efficiensies : & & & & & & \\
\hline & $\mathrm{SiH}_{4}=4, \mathrm{Si}_{2} \mathrm{H}_{6}=4$ & & & & & & \\
\hline \multirow[t]{7}{*}{6} & $\mathrm{Si}_{3} \mathrm{H}_{8}(+\mathrm{M})=\mathrm{SiH}_{4}+\mathrm{H}_{3} \mathrm{SiSiH}(+\mathrm{M})$ & & & & & & \\
\hline & High-pressure limit & $3.73 \mathrm{E}+12$ & 1 & 50850 & & & \\
\hline & Low-pressure limit & $4.36 \mathrm{E}+76$ & -17 & 59303 & & & \\
\hline & Troe parameters : & & & & & & \\
\hline & $a=0.4157, T^{* * *}=365.3, T^{*}=3102.0$ & & & & & & \\
\hline & Enhanced third-body efficiensies : & & & & & & \\
\hline & $\mathrm{SiH}_{4}=4, \mathrm{Si}_{2} \mathrm{H}_{6}=4$ & & & & & & \\
\hline \multirow[t]{7}{*}{7} & $\mathrm{H}_{3} \mathrm{SiSiH}(+\mathrm{M})=\mathrm{H}_{2} \mathrm{SiSiH}_{2}(+\mathrm{M})$ & & & & & & \\
\hline & High-pressure limit & $2.54 \mathrm{E}+13$ & -0.2 & 5381 & & & \\
\hline & Low-pressure limit & $1.10 \mathrm{E}+33$ & -5.8 & 9152 & & & \\
\hline & Troe parameters : & & & & & & \\
\hline & $a=-0.4202, T^{* * *}=214.5, T^{*}=103.0$ & & & & & & \\
\hline & Enhanced third-body efficiensies : & & & & & & \\
\hline & $\mathrm{SiH}_{4}=4, \mathrm{Si}_{2} \mathrm{H}_{6}=4$ & & & & & & \\
\hline
\end{tabular}


$\mathrm{H}_{3} \mathrm{SiSiH}_{+} \mathrm{SiH}_{4}(+\mathrm{M})=\mathrm{Si}_{2} \mathrm{H}_{6}+\mathrm{SiH}_{2}(+\mathrm{M})$

$1.73 \mathrm{E}+14 \quad 0.4$

8898.7

Reverse

$\begin{array}{lll}2.65 \mathrm{E}+15 & 0.1 & 8473.4\end{array}$

9

$\mathrm{H}_{3} \mathrm{SiSiH}(+\mathrm{M})=\mathrm{Si}+\mathrm{SiH}_{4}(+\mathrm{M})$

High-pressure limit

$1.42 \mathrm{E}+13 \quad 0.54 \quad 57548$

Low-pressure limit

$2.35 \mathrm{E}+4$

$-7.4$

60957

Troe parameters :

$a=0.5336, T^{* * *}=629.2, T^{*}=2190.0, T^{* *}=626.5$

Enhanced third-body efficiensies :

$\mathrm{SiH}_{4}=4, \mathrm{Si}_{2} \mathrm{H}_{6}=4$

\begin{tabular}{|c|c|c|c|c|}
\hline 10 & $\mathrm{Si}+\mathrm{Si}_{2} \mathrm{H}_{6}=\mathrm{SiH}_{2}+\mathrm{H}_{3} \mathrm{SiSiH}$ & $1.30 \mathrm{E}+15$ & 0 & 12600 \\
\hline 11 & $\mathrm{Si}_{3} \mathrm{H}_{8}=\mathrm{Si}_{3} \mathrm{H}_{6} \mathrm{~B}+\mathrm{H}_{2}$ & $2.00 \mathrm{E}+15$ & 0 & 56890 \\
\hline 12 & $\mathrm{Si}_{3} \mathrm{H}_{6}=\mathrm{Si}_{3} \mathrm{H}_{4} \mathrm{~B}+\mathrm{H}_{2}$ & $2.00 \mathrm{E}+15$ & 0 & 51700 \\
\hline 13 & $\mathrm{Si}_{4} \mathrm{H}_{10}=\mathrm{Si}_{4} \mathrm{H}_{8} \mathrm{~B}+\mathrm{H}_{2}$ & $2.00 \mathrm{E}+15$ & 0 & 54090 \\
\hline 14 & $\mathrm{Si}_{4} \mathrm{H}_{8}=\mathrm{Si}_{4} \mathrm{H}_{6} \mathrm{~B}+\mathrm{H}_{2}$ & $2.00 \mathrm{E}+15$ & 0 & 55070 \\
\hline 15 & $\mathrm{Si}_{5} \mathrm{H}_{12}=\mathrm{Si}_{5} \mathrm{H}_{10} \mathrm{~B}+\mathrm{H}_{2}$ & $2.00 \mathrm{E}+15$ & 0 & 54200 \\
\hline 16 & $\mathrm{Si}_{5} \mathrm{H}_{10}=\mathrm{Si}_{5} \mathrm{H}_{8} \mathrm{~B}+\mathrm{H}_{2}$ & $2.00 \mathrm{E}+15$ & 0 & 55300 \\
\hline 17 & $\mathrm{Si}_{5} \mathrm{H}_{8}=\mathrm{Si}_{5} \mathrm{H}_{6} \mathrm{~B}+\mathrm{H}_{2}$ & $2.00 \mathrm{E}+15$ & 0 & 43940 \\
\hline 18 & $\mathrm{Si}_{4} \mathrm{H}_{10}=\mathrm{Si}_{3} \mathrm{H}_{8}+\mathrm{SiH}_{2}$ & $2.00 \mathrm{E}+15$ & 0 & 55300 \\
\hline 19 & $\mathrm{Si}_{4} \mathrm{H}_{8}=\mathrm{Si}_{3} \mathrm{H}_{6}+\mathrm{SiH}_{2}$ & $2.00 \mathrm{E}+15$ & 0 & 73190 \\
\hline 20 & $\mathrm{Si}_{5} \mathrm{H}_{12}=\mathrm{Si}_{4} \mathrm{H}_{10}+\mathrm{SiH}_{2}$ & $2.00 \mathrm{E}+15$ & 0 & 57480 \\
\hline 21 & $\mathrm{Si}_{5} \mathrm{H}_{10}=\mathrm{Si}_{4} \mathrm{H}_{8}+\mathrm{SiH}_{2}$ & $2.00 \mathrm{E}+15$ & 0 & 65640 \\
\hline 22 & $\mathrm{Si}_{4} \mathrm{H}_{10}=\mathrm{Si}_{3} \mathrm{H}_{6} \mathrm{~B}+\mathrm{SiH}_{4}$ & $2.00 \mathrm{E}+15$ & 0 & 54490 \\
\hline 23 & $\mathrm{Si}_{4} \mathrm{H}_{8}=\mathrm{Si}_{3} \mathrm{H}_{4} \mathrm{~B}+\mathrm{SiH}_{4}$ & $2.00 \mathrm{E}+15$ & 0 & 67200 \\
\hline 24 & $\mathrm{Si}_{5} \mathrm{H}_{12}=\mathrm{Si}_{4} \mathrm{H}_{8} \mathrm{~B}+\mathrm{SiH}_{4}$ & $2.00 \mathrm{E}+15$ & 0 & 53870 \\
\hline 25 & $\mathrm{Si}_{5} \mathrm{H}_{10}=\mathrm{Si}_{4} \mathrm{H}_{6} \mathrm{~B}+\mathrm{SiH}_{4}$ & $2.00 \mathrm{E}+15$ & 0 & 63020 \\
\hline 26 & $\mathrm{Si}_{3} \mathrm{H}_{6} \mathrm{~B}=\mathrm{Si}_{3} \mathrm{H}_{6} \mathrm{~A}$ & $1.00 \mathrm{E}+13$ & 0 & 7500 \\
\hline 27 & $\mathrm{Si}_{3} \mathrm{H}_{4} \mathrm{~B}=\mathrm{Si}_{3} \mathrm{H}_{4} \mathrm{~A}$ & $1.00 \mathrm{E}+13$ & 0 & 7500 \\
\hline 28 & $\mathrm{Si}_{4} \mathrm{H}_{8} \mathrm{~B}=\mathrm{Si}_{4} \mathrm{H}_{8} \mathrm{~A}$ & $1.00 \mathrm{E}+13$ & 0 & 7500 \\
\hline 29 & $\mathrm{Si}_{4} \mathrm{H}_{6} \mathrm{~B}=\mathrm{Si}_{4} \mathrm{H}_{6} \mathrm{~A}$ & $1.00 \mathrm{E}+13$ & 0 & 7500 \\
\hline 30 & $\mathrm{Si}_{5} \mathrm{H}_{10} \mathrm{~B}=\mathrm{Si}_{5} \mathrm{H}_{10} \mathrm{~A}$ & $1.00 \mathrm{E}+13$ & 0 & 7500 \\
\hline 31 & $\mathrm{Si}_{5} \mathrm{H}_{8} \mathrm{~B}=\mathrm{Si}_{5} \mathrm{H}_{8} \mathrm{~A}$ & $1.00 \mathrm{E}+13$ & 0 & 7500 \\
\hline 32 & $\mathrm{Si}_{5} \mathrm{H}_{6} \mathrm{~B}=\mathrm{Si}_{5} \mathrm{H}_{6} \mathrm{~A}$ & $1.00 \mathrm{E}+13$ & 0 & 7500 \\
\hline 33 & $\mathrm{Si}_{3} \mathrm{H}_{6}=\mathrm{Si}_{3} \mathrm{H}_{6} \mathrm{~B}$ & $2.00 \mathrm{E}+15$ & 0 & 22140 \\
\hline 34 & $\mathrm{Si}_{4} \mathrm{H}_{8}=\mathrm{Si}_{4} \mathrm{H}_{8} \mathrm{~B}$ & $2.00 \mathrm{E}+15$ & 0 & 37230 \\
\hline 35 & $\mathrm{Si}_{5} \mathrm{H}_{10}=\mathrm{Si}_{5} \mathrm{H}_{10} \mathrm{~B}$ & $2.00 \mathrm{E}+15$ & 0 & 45500 \\
\hline 36 & $\mathrm{Si}_{5} \mathrm{H}_{8}=\mathrm{Si}_{5} \mathrm{H}_{8} \mathrm{~B}$ & $2.00 \mathrm{E}+15$ & 0 & 26360 \\
\hline 37 & $\mathrm{Si}_{4} \mathrm{H}_{10}=\mathrm{Si}_{2} \mathrm{H}_{4} \mathrm{~B}+\mathrm{Si}_{2} \mathrm{H}_{6}$ & $2.00 \mathrm{E}+15$ & 0 & 55230 \\
\hline 38 & $\mathrm{Si}_{5} \mathrm{H}_{12}=\mathrm{Si}_{3} \mathrm{H}_{6} \mathrm{~B}+\mathrm{Si}_{2} \mathrm{H}_{6}$ & $2.00 \mathrm{E}+15$ & 0 & 59610 \\
\hline
\end{tabular}




$\begin{array}{lllll}39 & \mathrm{Si}_{5} \mathrm{H}_{10}=\mathrm{Si}_{3} \mathrm{H}_{4} \mathrm{~B}+\mathrm{Si}_{2} \mathrm{H}_{6} & 2.00 \mathrm{E}+15 & 0 & 80480 \\ 40 & \mathrm{Si}_{5} \mathrm{H}_{12}=\mathrm{Si}_{3} \mathrm{H}_{8}+\mathrm{H}_{3} \mathrm{SiSiH} & 2.00 \mathrm{E}+15 & 0 & 60260 \\ 41 & \mathrm{Si}_{5} \mathrm{H}_{10}=\mathrm{Si}_{3} \mathrm{H}_{6}+\mathrm{H}_{3} \mathrm{SiSiH} & 2.00 \mathrm{E}+15 & 0 & 86320 \\ 42 & \mathrm{H}_{2} \mathrm{SiSiH}_{2}=\mathrm{SiH}_{2}+\mathrm{SiH}_{2} & 2.00 \mathrm{E}+15 & 0 & 61240 \\ 43 & \mathrm{Si}_{3} \mathrm{H}_{6} \mathrm{~A}=\mathrm{SiH}_{2}+\mathrm{H}_{2} \mathrm{SiSiH}_{2} & 2.00 \mathrm{E}+15 & 0 & 56410 \\ 44 & \mathrm{Si}_{4} \mathrm{H}_{8} \mathrm{~A}=\mathrm{H}_{3} \mathrm{SiSiH}_{2} \mathrm{H}_{2} \mathrm{SiSiH}_{2} & 2.00 \mathrm{E}+15 & 0 & 61470 \\ 45 & \mathrm{Si}_{5} \mathrm{H}_{10} \mathrm{~A}=\mathrm{Si}_{3} \mathrm{H}_{6} \mathrm{~B}+\mathrm{H}_{2} \mathrm{SiSiH}_{2} & 2.00 \mathrm{E}+15 & 0 & 64150 \\ 46 & \mathrm{Si}_{5} \mathrm{H}_{8} \mathrm{~A}=\mathrm{Si}_{3} \mathrm{H}_{4} \mathrm{~B}+\mathrm{H}_{2} \mathrm{SiSiH}_{2} & 2.00 \mathrm{E}+15 & 0 & 87010 \\ 47 & \mathrm{Si}_{5} \mathrm{H}_{10} \mathrm{~A}=\mathrm{H}_{3} \mathrm{SiSiH}+\mathrm{Si}_{3} \mathrm{H}_{6} \mathrm{~A} & 2.00 \mathrm{E}+15 & 0 & 60840 \\ 48 & \mathrm{Si}_{5} \mathrm{H}_{8} \mathrm{~A}=\mathrm{H}_{3} \mathrm{SiSiH}+\mathrm{Si}_{3} \mathrm{H}_{4} \mathrm{~A} & 2.00 \mathrm{E}+15 & 0 & 91030\end{array}$

a Reaction No. $1-3$ are pressure dependent disilane dissociation rate constants proposed by Matsumoto et al. ${ }^{7}$ (the same as TABLE 1) Reaction No. 4 - 10 are rate constants proposed by Girshick et al. ${ }^{27}$ (No. $4-7$ and No. 9 are expressed by the Troe parameters.) Reaction No.11 - 48 are rate constants of chemical species including more than three silicon atoms. $A$ factors are the same as those proposed by Girshick et al. ${ }^{27}$ and $E a(\Delta H)$ are calculated at the $\mathrm{HF} / 6-31 \mathrm{G}^{* *}$ level. Silenes are denoted as a suffix 'A' and silylenes are denoted as a suffix 'B'.

$\mathrm{b}$ Rate parameters are given in the units of molecule, $\mathrm{cm}$, s, cal and $\mathrm{K}$. 
TABLE S2: Surface phase rate constants. ${ }^{\text {a }}$

\begin{tabular}{|c|c|c|c|c|c|}
\hline No. & Reaction & A & $\beta$ & $\mathrm{E}_{\mathrm{a}}$ & note \\
\hline 1 & $\mathrm{SiH}_{4}+2 \mathrm{Si}(\mathrm{S})=2 \mathrm{SiH}(\mathrm{S})+\mathrm{Si}(\mathrm{B})+\mathrm{H}_{2}$ & $8.39 \mathrm{E}+26$ & 0 & 37450 & \\
\hline 2 & $\mathrm{Si}_{2} \mathrm{H}_{6}+2 \mathrm{Si}(\mathrm{S})=2 \mathrm{SiH}(\mathrm{S})+2 \mathrm{Si}(\mathrm{B})+2 \mathrm{H}_{2}$ & $8.39 \mathrm{E}+27$ & 0 & 37450 & \\
\hline 3 & $\mathrm{Si}_{3} \mathrm{H}_{8}+2 \mathrm{Si}(\mathrm{S})=2 \mathrm{SiH}(\mathrm{S})+3 \mathrm{Si}(\mathrm{B})+3 \mathrm{H}_{2}$ & $8.39 \mathrm{E}+27$ & 0 & 37450 & \\
\hline 4 & $\mathrm{Si}_{3} \mathrm{H}_{6}+2 \mathrm{Si}(\mathrm{S})=2 \mathrm{SiH}(\mathrm{S})+3 \mathrm{Si}(\mathrm{B})+2 \mathrm{H}_{2}$ & $8.39 \mathrm{E}+27$ & 0 & 37450 & \\
\hline 5 & $\mathrm{Si}_{4} \mathrm{H}_{10}+2 \mathrm{Si}(\mathrm{S})=2 \mathrm{SiH}(\mathrm{S})+4 \mathrm{Si}(\mathrm{B})+4 \mathrm{H}_{2}$ & $8.39 \mathrm{E}+27$ & 0 & 37450 & \\
\hline 6 & $\mathrm{Si}_{4} \mathrm{H}_{8}+2 \mathrm{Si}(\mathrm{S})=2 \mathrm{SiH}(\mathrm{S})+4 \mathrm{Si}(\mathrm{B})+3 \mathrm{H}_{2}$ & $8.39 \mathrm{E}+27$ & 0 & 37450 & \\
\hline 7 & $\mathrm{Si}_{5} \mathrm{H}_{12}+2 \mathrm{Si}(\mathrm{S})=2 \mathrm{SiH}(\mathrm{S})+5 \mathrm{Si}(\mathrm{B})+5 \mathrm{H}_{2}$ & $8.39 \mathrm{E}+27$ & 0 & 37450 & \\
\hline 8 & $\mathrm{Si}_{5} \mathrm{H}_{10}+2 \mathrm{Si}(\mathrm{S})=2 \mathrm{SiH}(\mathrm{S})+5 \mathrm{Si}(\mathrm{B})+4 \mathrm{H}_{2}$ & $8.39 \mathrm{E}+27$ & 0 & 37450 & \\
\hline 9 & $\mathrm{Si}_{5} \mathrm{H}_{8}+2 \mathrm{Si}(\mathrm{S})=2 \mathrm{SiH}(\mathrm{S})+5 \mathrm{Si}(\mathrm{B})+3 \mathrm{H}_{2}$ & $8.39 \mathrm{E}+27$ & 0 & 37450 & \\
\hline 10 & $\mathrm{SiH}(\mathrm{S})=\mathrm{Si}(\mathrm{S})+0.5 \mathrm{H}_{2}$ & $2.20 \mathrm{E}+11$ & 0 & 43300 & \\
\hline 11 & $\mathrm{Si}=\mathrm{Si}(\mathrm{B})$ & 1 & 0 & 0 & sticking coefficient \\
\hline 12 & $\mathrm{SiH}_{2}=\mathrm{Si}(\mathrm{B})+\mathrm{H}_{2}$ & 1 & 0 & 0 & sticking coefficient \\
\hline 13 & $\mathrm{H}_{2} \mathrm{SiSiH}_{2}=2 \mathrm{Si}(\mathrm{B})+2 \mathrm{H}_{2}$ & 1 & 0 & 0 & sticking coefficient \\
\hline 14 & $\mathrm{H}_{3} \mathrm{SiSiH}=2 \mathrm{Si}(\mathrm{B})+2 \mathrm{H}_{2}$ & 1 & 0 & 0 & sticking coefficient \\
\hline 15 & $\mathrm{Si}_{3} \mathrm{H}_{6} \mathrm{~A}=3 \mathrm{Si}(\mathrm{B})+3 \mathrm{H}_{2}$ & 1 & 0 & 0 & sticking coefficient \\
\hline 16 & $\mathrm{Si}_{3} \mathrm{H}_{6} \mathrm{~B}=3 \mathrm{Si}(\mathrm{B})+3 \mathrm{H}_{2}$ & 1 & 0 & 0 & sticking coefficient \\
\hline 17 & $\mathrm{Si}_{3} \mathrm{H}_{4} \mathrm{~A}=3 \mathrm{Si}(\mathrm{B})+2 \mathrm{H}_{2}$ & 1 & 0 & 0 & sticking coefficient \\
\hline 18 & $\mathrm{Si}_{3} \mathrm{H}_{4} \mathrm{~B}=3 \mathrm{Si}(\mathrm{B})+2 \mathrm{H}_{2}$ & 1 & 0 & 0 & sticking coefficient \\
\hline 19 & $\mathrm{Si}_{4} \mathrm{H}_{8} \mathrm{~A}=4 \mathrm{Si}(\mathrm{B})+4 \mathrm{H}_{2}$ & 1 & 0 & 0 & sticking coefficient \\
\hline 20 & $\mathrm{Si}_{4} \mathrm{H}_{8} \mathrm{~B}=4 \mathrm{Si}(\mathrm{B})+4 \mathrm{H}_{2}$ & 1 & 0 & 0 & sticking coefficient \\
\hline 21 & $\mathrm{Si}_{4} \mathrm{H}_{6} \mathrm{~A}=4 \mathrm{Si}(\mathrm{B})+3 \mathrm{H}_{2}$ & 1 & 0 & 0 & sticking coefficient \\
\hline 22 & $\mathrm{Si}_{4} \mathrm{H}_{6} \mathrm{~B}=4 \mathrm{Si}(\mathrm{B})+3 \mathrm{H}_{2}$ & 1 & 0 & 0 & sticking coefficient \\
\hline 23 & $\mathrm{Si}_{5} \mathrm{H}_{10} \mathrm{~A}=5 \mathrm{Si}(\mathrm{B})+5 \mathrm{H}_{2}$ & 1 & 0 & 0 & sticking coefficient \\
\hline 24 & $\mathrm{Si}_{5} \mathrm{H}_{10} \mathrm{~B}=5 \mathrm{Si}(\mathrm{B})+5 \mathrm{H}_{2}$ & 1 & 0 & 0 & sticking coefficient \\
\hline 25 & $\mathrm{Si}_{5} \mathrm{H}_{8} \mathrm{~A}=5 \mathrm{Si}(\mathrm{B})+4 \mathrm{H}_{2}$ & 1 & 0 & 0 & sticking coefficient \\
\hline 26 & $\mathrm{Si}_{5} \mathrm{H}_{8} \mathrm{~B}=5 \mathrm{Si}(\mathrm{B})+4 \mathrm{H}_{2}$ & 1 & 0 & 0 & sticking coefficient \\
\hline 27 & $\mathrm{Si}_{5} \mathrm{H}_{6} \mathrm{~A}=5 \mathrm{Si}(\mathrm{B})+3 \mathrm{H}_{2}$ & 1 & 0 & 0 & sticking coefficient \\
\hline 28 & $\mathrm{Si}_{5} \mathrm{H}_{6} \mathrm{~B}=5 \mathrm{Si}(\mathrm{B})+3 \mathrm{H}_{2}$ & 1 & 0 & 0 & sticking coefficient \\
\hline
\end{tabular}

a Reaction No.1 - 9 are temperature dependent sticking coefficients for silanes proposed by Ho et al. ${ }^{32} \mathrm{Si}(\mathrm{S})$ corresponds to a surface $\mathrm{Si}, \mathrm{SiH}(\mathrm{S})$ corresponds to a surface $\mathrm{SiH}$ and $\mathrm{Si}(\mathrm{B})$ corresponds to a bulk Si. Reaction No.10 is a hydrogen desorption reaction rate constant proposed by Sinniah et al. ${ }^{34}$

Reaction No.11 - 28 are sticking coefficients for $\mathrm{Si}$, silenes and silylenes proposed by Ho et al. ${ }^{32}$ Silenes are denoted as a suffix 'A' and silylenes are denoted as a suffix 'B'. 
TABLE S3: Concentrations of $\mathrm{Si}_{2} \mathrm{H}_{6}$ and $\mathrm{Si}_{3} \mathrm{H}_{8}$ measured at $\mathrm{T}=720 \mathrm{~K}$ and $\mathrm{P}=36.5$ Torr. ${ }^{\mathrm{a}}$ Concentration of $\mathrm{Si}_{2} \mathrm{H}_{4}$ are offset to the simulation value $\left(1.16 \times 10^{11}\right.$ molecule $\left.\mathrm{cm}^{-3}\right)$ at $\mathrm{t}=0.1 \mathrm{~s}$.

\begin{tabular}{cccc}
\hline reaction time $(\mathrm{s})$ & {$\left[\mathrm{Si}_{2} \mathrm{H}_{6}\right]$} & {$\left[\mathrm{Si}_{3} \mathrm{H}_{8}\right]$} & {$\left[\mathrm{Si}_{2} \mathrm{H}_{4}\right]$} \\
\hline 0.04 & $9.94 \mathrm{E}+14$ & $7.34 \mathrm{E}+12$ & $1.09 \mathrm{E}+11$ \\
0.05 & $9.86 \mathrm{E}+14$ & $6.32 \mathrm{E}+12$ & $1.25 \mathrm{E}+11$ \\
0.07 & $9.59 \mathrm{E}+14$ & $1.04 \mathrm{E}+13$ & $1.19 \mathrm{E}+11$ \\
0.10 & $9.56 \mathrm{E}+14$ & $1.51 \mathrm{E}+13$ & $1.16 \mathrm{E}+11$ \\
0.12 & $9.63 \mathrm{E}+14$ & $1.83 \mathrm{E}+13$ & $1.26 \mathrm{E}+11$ \\
0.16 & $9.42 \mathrm{E}+14$ & $2.45 \mathrm{E}+13$ & $1.24 \mathrm{E}+11$ \\
0.21 & $9.24 \mathrm{E}+14$ & $3.04 \mathrm{E}+13$ & $1.31 \mathrm{E}+11$ \\
0.24 & $9.01 \mathrm{E}+14$ & $2.97 \mathrm{E}+13$ & $1.66 \mathrm{E}+11$ \\
0.29 & $8.48 \mathrm{E}+14$ & $3.31 \mathrm{E}+13$ & $1.72 \mathrm{E}+11$
\end{tabular}

a Concentrations are given in the unit of molecule $\mathrm{cm}^{-3}$. 
TABLE S4: Concentrations of $\mathrm{Si}_{2} \mathrm{H}_{6}, \mathrm{Si}_{3} \mathrm{H}_{8}, \mathrm{Si}_{2} \mathrm{H}_{4}, \mathrm{Si}_{4} \mathrm{H}_{10}$, and $\mathrm{SiH}_{4}$ and branching ratios for $\mathrm{SiH}_{4}$ to $\mathrm{Si}_{3} \mathrm{H}_{8}$ at $\mathrm{T}=$ $720 \mathrm{~K}$ and $\mathrm{P}=36.5$ Torr predicted by the MSG1 model. ${ }^{\mathrm{a}}$

\begin{tabular}{ccccccc}
\hline reaction time $(\mathrm{s})$ & \multicolumn{1}{l}{$\left[\mathrm{Si}_{2} \mathrm{H}_{6}\right]$} & {$\left[\mathrm{Si}_{3} \mathrm{H}_{8}\right]$} & {$\left[\mathrm{Si}_{2} \mathrm{H}_{4} \mathrm{~A}\right]^{\mathrm{b}}$} & \multicolumn{1}{l}{$\left[\mathrm{Si}_{4} \mathrm{H}_{10}\right]$} & \multicolumn{1}{l}{$\left[\mathrm{SiH}_{4}\right]$} & {$\left[\mathrm{SiH}_{4}\right] /\left[\mathrm{Si}_{3} \mathrm{H}_{8}\right]$} \\
\hline 0.01 & $1.00 \mathrm{E}+15$ & $6.11 \mathrm{E}+11$ & $3.39 \mathrm{E}+10$ & $4.71 \mathrm{E}+09$ & $8.25 \mathrm{E}+11$ & 1.35 \\
0.03 & $1.00 \mathrm{E}+15$ & $3.27 \mathrm{E}+12$ & $8.29 \mathrm{E}+10$ & $5.72 \mathrm{E}+10$ & $4.08 \mathrm{E}+12$ & 1.25 \\
0.04 & $9.97 \mathrm{E}+14$ & $4.52 \mathrm{E}+12$ & $8.92 \mathrm{E}+10$ & $9.02 \mathrm{E}+10$ & $5.67 \mathrm{E}+12$ & 1.25 \\
0.06 & $9.92 \mathrm{E}+14$ & $6.89 \mathrm{E}+12$ & $9.89 \mathrm{E}+10$ & $1.64 \mathrm{E}+11$ & $8.88 \mathrm{E}+12$ & 1.29 \\
0.10 & $9.80 \mathrm{E}+14$ & $1.14 \mathrm{E}+13$ & $1.16 \mathrm{E}+11$ & $3.40 \mathrm{E}+11$ & $1.53 \mathrm{E}+13$ & 1.35 \\
0.15 & $9.66 \mathrm{E}+14$ & $1.65 \mathrm{E}+13$ & $1.33 \mathrm{E}+11$ & $5.97 \mathrm{E}+11$ & $2.36 \mathrm{E}+13$ & 1.43 \\
0.20 & $9.52 \mathrm{E}+14$ & $2.13 \mathrm{E}+13$ & $1.43 \mathrm{E}+11$ & $8.78 \mathrm{E}+11$ & $3.21 \mathrm{E}+13$ & 1.51 \\
0.25 & $9.38 \mathrm{E}+14$ & $2.57 \mathrm{E}+13$ & $1.50 \mathrm{E}+11$ & $1.17 \mathrm{E}+12$ & $4.06 \mathrm{E}+13$ & 1.58 \\
0.30 & $9.24 \mathrm{E}+14$ & $2.99 \mathrm{E}+13$ & $1.54 \mathrm{E}+11$ & $1.47 \mathrm{E}+12$ & $4.90 \mathrm{E}+13$ & 1.64 \\
0.35 & $9.11 \mathrm{E}+14$ & $3.39 \mathrm{E}+13$ & $1.56 \mathrm{E}+11$ & $1.78 \mathrm{E}+12$ & $5.77 \mathrm{E}+13$ & 1.70 \\
0.40 & $8.97 \mathrm{E}+14$ & $3.76 \mathrm{E}+13$ & $1.57 \mathrm{E}+11$ & $2.08 \mathrm{E}+12$ & $6.64 \mathrm{E}+13$ & 1.76
\end{tabular}

a Concentrations are given in the unit of molecule $\mathrm{cm}^{-3}$.

b Silene $\left(\mathrm{H}_{2} \mathrm{SiSiH}_{2}\right)$. 